\title{
非线性极大极小问题的一个有效解法 ${ }^{*}$ \\ 李兴斯 \\ (大连理工大学工程力学研究所,大连 116024)
}

\section{关线词极大极小、不可微优化、最大摘原理}

一个非线性极大极小问题 (A)通常表达为

$$
\underset{x}{\operatorname{minimize}} \varphi(x)-\max _{i}\left\{f_{i}(x)\right\},
$$

式中 $f_{i}(x)$ 一般为变量 $x \in \mathrm{R}^{*}$ 的光滑非线性函数, $i=1, \cdots, m$. 由于目标函数 $\varphi(x)$ 是不可 微的, 故 (A) 是一个不可微的无约束优化问题, 因此不能使用标准的无约束优化算法求解, 通 常将其化为下述等价的非线性规划问题 (B):

$$
\begin{aligned}
& \underset{x, z}{\operatorname{minimize}} z, \\
& \text { subject to } g_{i}(x, z)-f_{i}(x)-z \leqslant 0, i-1, \cdots, m,
\end{aligned}
$$

其中 $z$ 是一个新引进的独立变量. 这种变换虽然能将不可微问题转化成可微问题, 但却把原 来的无约束问题变成了有约束问题,失去了无约束的优点.

本文用 Jaynes ${ }^{[2]}$ 提出的最大嫡原理作为一个辅助推理准则, 导出一个可微函数 $F_{p}(x)$, 借以直接通近 $\varphi(x)$, 从而使得到的问题既可微又保持无约束的优点. 采用本文方法, 问题 (A)的求解被转化为如下无约束优化问题 (C):

$$
\underset{x}{\operatorname{minimize}} F_{p}(x)-\frac{1}{p} \ln \left\{\sum_{i=1}^{m} \exp \left[p f_{i}(x)\right]\right\},
$$

其中 $p$ 是一个控制参数. 可以证明, 当参数 $P$ 趋于无穷大时, 函数 $F,(x)$ 在整个变量空间一致 逼近函数 $\varphi(x)$. 实际计算时, $p$ 可取一比较大的有限值, 只用一次无约束优化计算, 就可得到 原问题足够精确的解.

下面我们给出函数 $F,(x)$ 的推导过程. 众所周知, 极大极小问题 (A)的 Lagrange 函数为

$$
L(x, \lambda)=\sum_{i=1}^{m} \lambda_{i} f_{i}(x),
$$

其中的 Lagrange 乘子 $\lambda$ 属于单纯形集合

$$
\Lambda-\left(\lambda \geqslant 0: \sum_{i=1}^{m} \lambda_{i}=1\right),
$$

由此可见, Lagrange 函数 $L(x, \lambda)$ 相当于问题 $(\mathrm{A})$ 中诸函数的凸组合. 在适当条件下,该函 数满足下述关系:

$$
\max _{\lambda} \min _{x} L(x, \lambda)-\min _{x} \max _{\lambda} L(x, \lambda),
$$

本文 1990 年 12 月4 日收到. 1991 年5月9日收到修改稿.

- 国家自然科学美金资助项目. 
上述等式的左边对应“原-对偶”方法, 即先求函数 $L(x, \lambda)$ 关于 $x$ 的极小解 $x(\lambda)$, 再求其对 偶函数 $L(x(\lambda), \lambda)$ 关于 $\lambda$ 的极大解. 在一般情况下,极小解 $x(\lambda)$ 没有解析表达式,整个求 解过程需在原与对偶空间交叉迭代, 解法的收玫速度很慢. 与这种原一对偶法相反, 我们将采 用 “对偶一原”方法,先求 $L(x, \lambda)$ 关于 $\lambda$ 的极大解 $\lambda(x)$, 再求 $L(x, \lambda(x))$ 关于 $x$ 的极小解; 即需先解如下关于 $\lambda$ 的极大化问题 (D):

$$
\underset{\lambda \in A}{\operatorname{maximize}} L(x, \lambda)=\sum_{i=1}^{m} \lambda_{i} f_{i}(x) .
$$

然而, 直接求解上述问题得不到解析解, 为此我们引进最大嫡原理. 因为 $L(x, \lambda)$ 相当于 问题(A)中诸函数的凸组合, 故可将 Lagrange 乘子 $\lambda_{i}$ 解释为相应函数 $f_{i}(x)$ 在解点等于最 大值函数 $\varphi(x)$ 的“概率”, 这样 Lagrange 函数可视为问题 (A)中诸函数的“均值”; 并且, 问题 (D)的求解过程可以解释为寻求一个概率分布函数 $\lambda(\boldsymbol{x})$. 按照最大摘原理的要求, 这个概率 分配应当使 Shannon ${ }^{[2]}$ 摘函数达到最大值, 我们把这个关于摘函数极大化的要求作为一个“辅 助”极大化目标, 构造一个“复合的”极大化问题 $(E)$ :

$$
\underset{x \in A}{\operatorname{maximize}} L_{p}(x, \lambda)=L(x, \lambda)+H(\lambda) / p \text {, }
$$

工虫 $H(\lambda)$ 为 Shannon 摘, 其值由下式给出:

$$
H(\lambda)=-\sum_{i=1}^{m} \lambda_{i} \ln \lambda_{i}
$$

容易看出, 问题( $E$ )中实际包含两个极大化的目标, 一个是问题 (D) 中的目标函数 $L(x, \lambda)$, 另 一个则是由最大嫡原理提供的摘函数 $H(\lambda)$, 参数 $p$ 相当于两个目标函数间的加权系数. 显 然, 当 $p$ 趋于无穷大时, 问题(E)将退化为问题(D), 因为嫎函数 $H(\lambda)$ 总取一个非负的有限 值. 我们构造这个双目标问题的主要原因在于,通过简单的计算,即可得到该问题的一个解析 解:

$$
\lambda_{i}(x)-\exp \left[p f_{i}(x)\right] / \sum_{i=1}^{m} \exp \left[p f_{i}(x)\right], i-1, \cdots, m,
$$

将其回代到问题 $(E)$ 中的目标函数 $L_{p}(x, \lambda)$, 消去 $\lambda$, 即可得到一个仅为变最 $x$ 的函数, 该函 数就是由(4)式给出的无约束优化问题的目标函数 $F_{p}(x)$.

关于函数 $F_{p}(x)$ 的性质, 有如下诸定理, 证明从略.

定理 $1 \forall \lambda \in \Lambda$, 均成立不等式:

$$
F_{p}(x) \geqslant L_{p}(x, \lambda) \text {. }
$$

定理 $2 F_{p}(x)$ 随 $P$ 的增加单调下降; 且当 $P$ 趋于无穷大时, $F_{p}(x)$ 趋于 $\varphi(x)$.

定理 $3 \forall x \in \mathrm{R}^{*}, F_{p}(x)$ 与 $\varphi(x)$ 间皆满足下列不等式:

$$
\varphi(x) \leqslant F_{p}(x) \leqslant \varphi(x)+\ln (m) / p \text {. }
$$

由上述定理可见, $F_{p}(x)$ 由上方逼近 $\varphi(x)$; 且当 $p$ 取得充分大时, 两者之差可忽略不计. 因 此实际计算时, $p$ 可视问题精度要求取一适当的有限值.

善本茾法 1. 令 $P$ 为一个充分大常数, 给 $x$ 䟼初值；2. 用无约束优化算法的子程序进 行 $F_{p}(x)$ 的最小化计算.

值策例及计策结果例题取自文献[3]. 计算中使用一个标准的 DFP 算法子程序; $P$ 取为 $10^{6}$, 向量 $x$ 的初值均为 0 ; 计算结果列于表 1 .

a $1 f_{1}(x)-x_{1}^{2}+x_{2}^{4}, f_{2}(x)-\left(2-x_{1}\right)^{2}+\left(2-x_{2}\right)^{2}, f_{3}(x)-2 \exp \left(-x_{1}+x_{2}\right)$. 
(i) $2 \quad f_{1}(x)=x_{1}^{4}+x_{2}^{2}, f_{2}(x)-\left(2-x_{1}\right)^{2}+\left(2-x_{2}\right)^{2}, f_{3}(x)-2 \exp \left(-x_{1}+x_{2}\right)$.

表 1 例题计算结果

\begin{tabular}{|c|c|c|c|}
\hline 昌号 & 解的来源 & $x$ & $f_{i}(x)$ \\
\hline \multirow{2}{*}{1} & 最优解 & $1.13904,0.89956$ & $1.95222,1.95222,1.57408$ \\
\hline & 本文解 & $1.13903774,0.89955982$ & $1.95222445,1.95222453,1.57407731$ \\
\hline \multirow{2}{*}{2} & 最优魚 & 1,1 & $2,2,2$ \\
\hline & 本文解 & $1.00000012,0.999999973$ & $1.99999976,2.00000041,1.99999925$ \\
\hline
\end{tabular}

事实上, 函数 $F_{p}(x)$ 也可由 $L$, 模的定义直接导出. 设有一向量值函数 $\psi(x)$, 其分量为

$$
\psi_{i}(x)=\exp \left[f_{i}(x)\right], i=1, \cdots, m .
$$

因所有分量为正, 故 $L$, 模可直接定义为

$$
\|\varphi(x)\|_{p}-\left\{\sum_{i=1}^{m}\left[\psi_{i}(x)\right]^{p}\right\}^{1 / p}-\left\{\sum_{i=1}^{m} \exp \left[p f_{i}(x)\right]\right\}^{1 / p},
$$

其中 $p \geqslant 1$. 上式两边取自然对数,即得到

$$
\ln \|\psi(x)\|_{p}-(1 / p) \ln \left\{\sum_{i=1}^{m} \exp \left[p f_{i}(x)\right]\right\}=F_{p}(x) .
$$

由此看出, 函数 $F_{p}(x)$ 原来相当于诸函数 $f_{i}(x)$ 的指数函数为分量构成的向量函数 $\psi(x)$ 的 $L_{p}$ 模的自然对数. 其中的指数变换使向量的各分量为正, 而对数变换又使函数值复原. 尽管从 数学上说, 这个推导可能易于接受, 但由最大嫡原理给出的推导, 可加深对这个函数的理解, 同 时也可揭示 $L_{p}$ 模逼近过程的摘本质. 由于函数 $F,(x)$ 可由 $L_{p}$ 模的定义直接导出, 因此也 具有 $L$, 模的某些有用性质.

由前面给出的理论与数值结果可以看出, 本文提出的求解非线性极大极小问题的方法比 之其它解法具有许多特点. 首先, 从理论上可以加深对极大极小问题求解思想的认识; 其次就 是算法实现的简单性与计算的高效率. 采用本文方法, 可以直接利用现有的标准无约束优化 算法软件, 这为用户提供了很大方便. 极大极小问题在科学技术中具有厂泛的应用, 如数据拟 合、解不等式组及过定方程组等, 本文方法可直接推广到这些问题的求解.

致谢: 作者感谢大连理工大学钱令希教授和英国利物浦大学 A. B. Templeman 教授在工作中给予的 鼓励与帮助.

\section{考文献}

[1] Jaynes, E. T., Phy. Rev., 106 (1957), 620-630.

[2] Shannon, C. E., Bell. Sys. Tech. J., 27 (1948), 279-428.

[3] Charalambous, C. and Conn, A. R., SIAM J. Nwm. Anal., 15 (1978), 162-187. 